\title{
Molecular Identification of Polyhydroxyalkanoates-Producing Bacteria Isolated from Enriched Microbial Community
}

\author{
SŁAWOMIR CIESIELSKI*, TOMASZ POKOJ, JUSTYNA MOŻEJKO and EWA KLIMIUK
}

Department of Environmental Biotechnology, University of Warmia and Mazury in Olsztyn, Poland

Received 6 November 2011, revised 18 October 2012, accepted 18 October 2012

Abstract

Polyhydroxyalkanoates (PHAs) are especially interesting because of their similar properties to synthetic plastics and their potential use as biodegradable polymers. Many strategies have been employed to effectively and economically produce PHAs, among them a production process based on mixed microbial populations, enriched from activated sludge could be one of the alternative technologies. Defining the bacterial species creating these anonymous populations is crucial for the improvement of cultivation strategy. Moreover, enriched bacterial populations could be a promising source for microbes, useful in many biotechnological projects. The main object of this study was to characterize the microorganisms creating the microbial consortium cultured towards PHAs production. After cultivation, bacteria were identified using the 16S rRNA gene sequencing approach. The presence of genes engaged in PHAs synthesis was detected using PCR. The performed analysis revealed that among eleven isolated bacterial strains, four possessed the ability of polyhydroxybutyrate synthesis.

Key words: 16S rRNA, biopolymers, microbial community, PHB, polyhydroxyalkanoates

\section{Introduction}

Polyhydroxyalkanoates (PHAs) are the polymers of hydroxyalkanoates that accumulate as carbon/energy storage material synthesized by various microorganisms. PHAs have received much attention in recent times because of their biodegradable nature and properties similar to plastics from petrochemical products (Anderson and Dawes, 1990). Industrial production of PHAs is currently being conducted using bacterial cultures of pure or recombinant strains that require sterile production conditions and strict process control. This state of the art technology has a high energy demand and, consequently, high production costs relative to those of, for example, polypropylene. Furthermore, well-defined and expensive substrates, such as glucose or propionic acid, are used as feedstock, contributing even further to the relatively high costs of production (Pisco et al., 2009). An attractive alternative to pure microbial cultures for the production of PHAs may be the use of mixed cultures, such as those associated with activated sludge from wastewater treatment plants (Satoh et al., 1998).

One of the main issues in PHAs production using the mixed culture processes is microorganism selection.
The mixed microbial culture used in these processes is enriched in PHAs-accumulating bacteria although other microorganisms are also present. Selection of a stable culture with a high PHAs storage capacity is crucial for the high efficiency of the process. The microorganisms involved experience rapidly changing conditions of nutrient availability and can adapt continuously to changes in the substrate concentration. Microorganisms which are able to quickly store the available substrate and next consume the storage materials to achieve a more balanced growth have a strong competitive advantage over microorganisms without this ability (Van Loosdrecht et al., 1997).

High substrate costs decrease the profitability of PHAs production, and thus low-cost carbon substrates such as agricultural and industrial residues or by-products have been tested for the production of these polyesters (Dias et al., 2006). Among them, crude glycerine, formed as a by-product during biodiesel production, seems to be a promising source of carbon (da Silva et al., 2009). Due to increased glycerol production by the growing biodiesel industry, the prices for glycerine have become low enough to use this residual compound as a cheap carbon source for several industrial fermentation processes, especially for the production

* Corresponding author: S. Ciesielski, Department of Environmental Biotechnology, ul. Słoneczna 45G, 10-709 Olsztyn, Poland; phone: (+48) 895234162; fax: (+48) 895234131; e-mail: slavcm@uwm.edu.pl 
of microbial polyesters (Ibrahim and Steinbüchel, 2009). Additionally, new strains, using economic substrates and having a high accumulation percentage, must be utilized to make the process of PHAs production economically reasonable. Various researches have isolated the promising microorganisms from different environment such as marine microbial mats (LópezCortés et al., 2008), date syrup waste (Ataei et al., 2008) the root nodules of leguminous plants (Kumbhakar et al., 2012). Prognosticating source of PHAs producing bacteria could be microbial communities developing during enrichment of the activated sludge (Ciesielski et al., 2006).

Therefore, the main goal of this work was identifying members of a mixed microbial culture enriched from activated sludge, which could potentially produce PHAs. Additionally, the process of PHAs synthesis by mixed microbial culture was characterized.

\section{Experimental}

\section{Materials and Methods}

Experiment set-up. Crude glycerine, obtained from Elstar Oils Company (Malbork, North Poland), was used as a carbon source. The culture medium was prepared as follows: $18 \mathrm{~g}$ (phase I) and $54 \mathrm{~g}$ (phase II) of crude glycerine was dissolved in one litre of distilled water and supplemented with a mineral salts solution according to Serafim et al. (2004).

The experiment was performed in a laboratory-scale sequencing batch reactor (SBR), with a working volume of 51, initially inoculated with activated sludge obtained from a municipal wastewater treatment plant in Olsztyn (North Poland). The SBR was operated under aerobic conditions in cycles of $24 \mathrm{~h}$. PHAs accumulation was favored by dividing the SBR cycle into three phases: phase I (biomass growth), phase II (PHAs synthesis under ammonia limited conditions) and phase III (PHAs consumption as an endogenous carbon source). This was attained by continuous feeding of the culture medium with a distinct chemical constitution.

In phase I culture medium consisted of crude glycerine and mineral salts solution. Organics and ammonia concentration were $21.1 \mathrm{~g} C O D / 1$ and $703.2 \mathrm{mg}$ $\mathrm{N}-\mathrm{NH}_{4} / 1$. The concentration of ammonia was adjusted, so neither limit the cell growth nor remain in the mixed liquor at the end of the phase. During phase II ammonia-free culture medium was fed and crude glycerine concentration was increased to $60.9 \mathrm{~g} \mathrm{COD} / \mathrm{l}$, whereas in phase III glycerine-free culture medium, containing only the mineral salts solution was supplied.

The duration of the following phases was 6, 6 and $12 \mathrm{~h}$, respectively. The volume of culture medium was
$0.25 \mathrm{l}$ in phase I, $0.25 \mathrm{l}$ in phase II, and $0.5 \mathrm{l}$ in phase III. PHAs-rich excess biomass was withdrawn from the SBR at the end of the PHAs synthesis phase (phase II). The volume of removed biomass was adjusted to maintain the concentration of biomass remaining in the reactor at $3 \mathrm{~g} \mathrm{c.d}$. w. $/ \mathrm{l}$ at the beginning of the next cycle, after its dilution by culture medium feeding in phase III. The concentration of $3 \mathrm{~g}$ c.d. w./l was assumed as typical value applied in activated sludge chamber in wastewater treatment plants and allowed to maintain complete mixing and adequate aeration of biomass (dissolved oxygen concentration of around $80 \%$ of saturation level).

Polyhydroxyalkanoates analysis. The fermentation was controlled by taking samples once per week. In order to determine PHAs concentration in a day, the samples were collected every hour from the beginning to $14^{\text {th }}$ hour and at the end of the SBR cycle. The analysis of PHAs was repeated four times. The PHAs in the biomass were determined by the procedures described by Comeau et al. (1988) and Oehmen et al. (2005) with a minor modification. Biomass samples taken from SBR were centrifuged immediately and the supernatant was decanted. The resulting pellet was collected and stored at $-70^{\circ} \mathrm{C}$ prior to further analysis. All samples were then lyophilized through a freeze drying unit (Lyovac GT2, Steris GmbH, Germany) operated for at least $20 \mathrm{~h}$. Next, $2 \mathrm{ml}$ of chloroform and $2 \mathrm{ml}$ of an acidified methanol solution (containing 3\% sulfuric acid by volume and benzoic acids as the internal standard) were added to the lyophilised biomass. In parallel with the biomass samples, a standard solution composing of a R-3-hydroxybutyric acid (3HB) and R-3-hydroxyvaleric acid $(3 \mathrm{HV})$ copolymer (12\% of $3 \mathrm{HV}$ ) (Sigma-Aldrich) was prepared. The samples and standard were then digested for $20 \mathrm{~h}$ at $100^{\circ} \mathrm{C}$, and cooled to room temperature. One milliliter of distilled water was then added to each sample and mixed vigorously to remove particulate debris from the chloroform phase. After mixing, $1 \mathrm{~h}$ of settling time was allowed to achieve phase separation. The chloroform (bottom) phase was dried with approximately $0.5-1 \mathrm{~g}$ of anhydrous sodium sulphate. One microlitre of the chloroform phase was then transferred to a vial and analyzed with a Varian gas chromatograph. The chromatograph was operated with a VF-5 ms column (30 m length $\times 0.25 \mathrm{~mm}$ I.D. $\times 0.25 \mu \mathrm{m}$ film, Varian), a split injection ratio of 1:15 and helium as the carrier gas $(1.0 \mathrm{ml} / \mathrm{min})$. The injection port temperature was $250^{\circ} \mathrm{C}$ and flame ionization detection (FID) unit $-300^{\circ} \mathrm{C}$.

Microbial isolation. Sludge samples taken from the bioreactors were washed with a $0.5 \% \mathrm{NaCl}$ solution by natural settling, and sludge flocks were then dispersed using a VCX 130PB Ultrasonicator for $30 \mathrm{~s}$ at $50 \mathrm{~W}$ (Sonics, Newtown, CT, U.S.A.). Sludge samples serially diluted with sterile water were plated onto GM1, LB, 
NBYB, and King B media. After 8 days of incubation at a temperature of $30^{\circ} \mathrm{C}$, one specimen of each colony morphotype was picked onto a fresh plate. Once the purity of each of these cultures had been verified, they were stored as glycerol stocks at $-80^{\circ} \mathrm{C}$. Cultivation in liquid medium was carried out in $15-\mathrm{ml} \mathrm{screw-capped}$ tubes with shaking $(200 \mathrm{rpm})$ at the temperature of $30^{\circ} \mathrm{C}$. After $20 \mathrm{~h}$, cultivation was stopped and $2 \mathrm{ml}$ of each bacterial strain was subjected to genetic analysis.

DNA extraction, amplification and sequencing. DNA extraction was performed as follows: $2 \mathrm{ml}$ of fresh bacterial culture was pelleted by centrifugation, suspended in the proteinase $\mathrm{K}$ buffer $(100 \mathrm{mM}$ Tris$\mathrm{HCl} ; 10 \mathrm{mM}$ EDTA; $\mathrm{pH} 8.0$ ), and incubated at $55^{\circ} \mathrm{C}$ in the presence of sodium dodecyl sulfate, proteinase $\mathrm{K}$, and lysozyme. DNA was purified using silica based columns (A\&A Biotechnology, Poland) and eluted with $100 \mu \mathrm{l}$ of TRIS/EDTA buffer ( $10 \mathrm{mM}$ Tris- $\mathrm{HCl}, 1 \mathrm{mM}$ EDTA, pH 7.5).

The partial 16S rRNA gene was amplified using the primers 8F: 5'-GTG CTG CAGAGA GTT TGA TCC TGG CTCAG-3' and 536R: 5'-CAC GGA TCC GTA TTA CCG CGG CTG CTG-3' described previously by Miskin et al. (1999). In order to detect microorganism producing polyhydroxyalkanoates, PCR primers: G-D: 5' GTG CCG CC(GC) (CT)(AG)(GC) ATC AAC AAG T-3' and G1-R: 5'-GTT CCA G(AT) ACA G(GC) A (GT)(AG) T CGA A-3') were applied (Romo et al., 2007). PCR was performed in an Eppendorf Mastercycler Gradient (Eppendorf, Germany). The mixtures used for PCR amplification contained $50 \mathrm{ng}$ of extracted total DNA, $0.5 \mu \mathrm{M}$ of each primer, $100 \mu \mathrm{M}$ of deoxynucleoside triphosphate (Promega, Wisconsin, U.S.A.), $1 \mathrm{U}$ of Taq DNA polymerase (Invitrogen, Life Technologies), $5 \mu \mathrm{l}$ of reaction buffer $(500 \mathrm{mM} \mathrm{KCl}$, $\mathrm{pH} 8.5$; Triton X-100), $1.5 \mathrm{mM} \mathrm{MgCl} 2$, and sterile water to a final volume of $50 \mu \mathrm{l}$. PCR amplification of the PHA synthase gene fragment was carried out using the program given by the authors. The temperature program for $16 \mathrm{~S}$ rRNA gene amplification was as follows: $94^{\circ} \mathrm{C}$ for $5 \mathrm{~min}$; 30 cycles of denaturation at $94^{\circ} \mathrm{C}$ for $30 \mathrm{~s}$, annealing at $54^{\circ} \mathrm{C}$ for $30 \mathrm{~s}$, extension at $72^{\circ} \mathrm{C}$ for $1 \mathrm{~min}$, and a single final elongation at $72^{\circ} \mathrm{C}$ for $5 \mathrm{~min}$. The PCR amplicons of PHA synthase genes were resolved on $1.0 \%$ agarose gels stained with ethidium bromide, and the size of the PCR products was estimated using molecular mass marker (100 bp; Promega, Wisconsin, U.S.A.). The sequencing of $16 \mathrm{~S}$ rRNA fragments was performed using a Perkin Elmer ABI 373 Automated DNA Sequencer (PE Applied Biosystems, Foster City, CA, U.S.A.) at a commercial company (Genomed, Warsaw, Poland). All reactions were run following the manufacturer's protocols. The nucleotide sequences were submitted to the GenBank database under accession numbers from HQ292693 to HQ292703.
Phylogenetic Analysis. The sequences of 16S rDNA genes were compared with those from the GenBank database using the NCBI Blast program. Sequences were aligned using the ClustalW program (Thompson et al., 1994). Genetic relationships were determined by the neighbor-joining method with the MEGA2 program (Kumar et al., 2001) using nucleotide sequences of the $16 \mathrm{~S}$ rDNA gene. To determine the degree of statistical support for branches in the phylogeny, 1,000 bootstrap replicates of data were analyzed. The gene sequences that were $>94.0 \%$ identical to sequences of species in the NCBI database were assigned genus names (Stackebrandt and Goebel, 1994).

\section{Results}

Profiles of PHAs concentration in mixed liquor and PHAs content in biomass are presented in Figure 1. The obtained results revealed that during the biomass growth phase (phase I), PHAs concentration ranged from 510.8 to $587.1 \mathrm{mg}$ PHA/l and did not vary significantly. The accumulation of PHAs began after the cessation of ammonia feeding with the culture medium
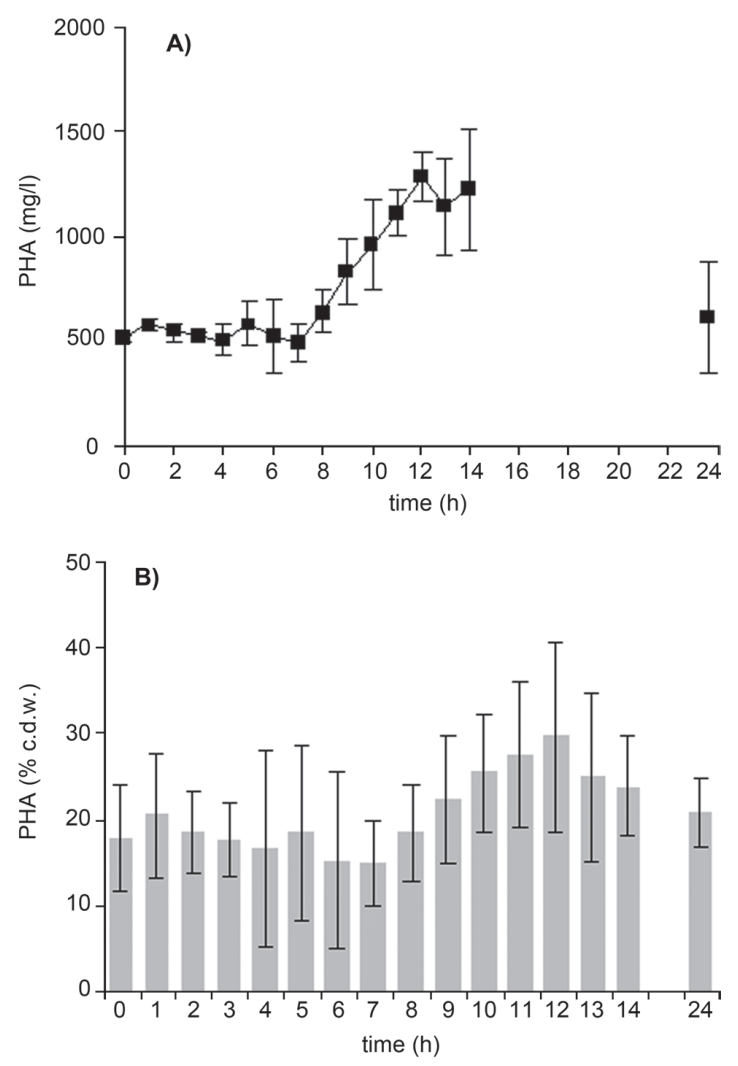

Fig. 1. Profiles of PHAs concentration in mixed liquor (A) and PHAs content (B) in biomass measured during 24 hours. Mean values were obtained from three measurements. 
(phase II). Polymer concentration increased linearly, reaching the highest value of $1285.43 \mathrm{mg}$ at $12 \mathrm{~h}$ of the cycle, which corresponded to $29.7 \%$ of cell dry weight. The observed PHA storage yield, calculated based on the mass of PHAs withdrawn from SBR with excess sludge and the mass of crude glycerine consumption (as COD), was $0.30 \mathrm{~g}$ PHA/g COD. In phase III, when crude glycerine feeding to the SBR was stopped, PHAs concentration decreased due to their consumption by the microorganisms as an endogenous carbon source. At the end of this phase, PHAs concentration amounted to $0.615 \mathrm{~g}$ PHA/l (20.6\% c.d.w.).

Samples taken from the bioreactor on day 162 of the experiment were serially diluted and plated on 4 different types of standard microbiological culture media. After 8 days of incubation, 20 morphologically different colonies originating from all the culture media were subjected to genetic analysis. Among the 20 isolates analyzed, eleven had a unique $16 \mathrm{~S}$ rRNA gene fragment. Afterwards, the isolates were assigned to a genus and species according to similarity with the $16 \mathrm{~S}$ rRNA sequences deposited in the NCBI GenBank. The result of phylogenetic analysis based on the almost $1000 \mathrm{bp}$ long $16 \mathrm{~S}$ rDNA sequences from the evolutionary distances created by the neighbor-joining method is shown in Fig. 2. The cultivable microorganisms were affiliated with six bacterial lineages: Alfaproteo-
Table I

Molecular identification of isolates possessing genetic potential of PHAs synthesis

\begin{tabular}{|l|l|c|}
\hline Isolate & $\begin{array}{c}\text { Organisms with closest 16S rRNA } \\
\text { gene sequence }\end{array}$ & $\begin{array}{c}\text { Coverage/ } \\
\text { identity (\%) }\end{array}$ \\
\hline G8_4 & Comamonas aquatica (EU 841527) & $100 / 100$ \\
\hline G8_19 & Bacillus thuringensis (GU 826152) & $100 / 100$ \\
\hline G8_22 & Caulobacter fusiformis (NR 025320) & $100 / 99$ \\
\hline G8_25 & Aeromonas hydrophila (FN 997617) & $100 / 99$ \\
\hline
\end{tabular}

bacteria, Betaproteobacteria, Gammaproteobacteria, Flavobacteria, Actinobacteria, and Bacilli. Three of the isolates were assigned to Gammaproteobacteria, among them two isolates (G8_2 and G8_9) were the most similar to the Citrobacter species, whereas one (G8_1) was closely related to Serratia marcescens. The strains G8_7 and G8_15 were classified as Microbacterium species (Actinobacteria). Alfaproteobacteria were represented by G8_28, which is closely related to the Sphingomonas species, and G8_22 affiliated with the Caulobacter species. The rest of the lineages were instanced by single isolates. Betaproteobacteria were represented by G8_4, which was most similar to the Comamonas species, Bacilla were exemplified by G8_19 (Bacillus sp.), whereas isolate G8_24 belonged to Flavobacteria.

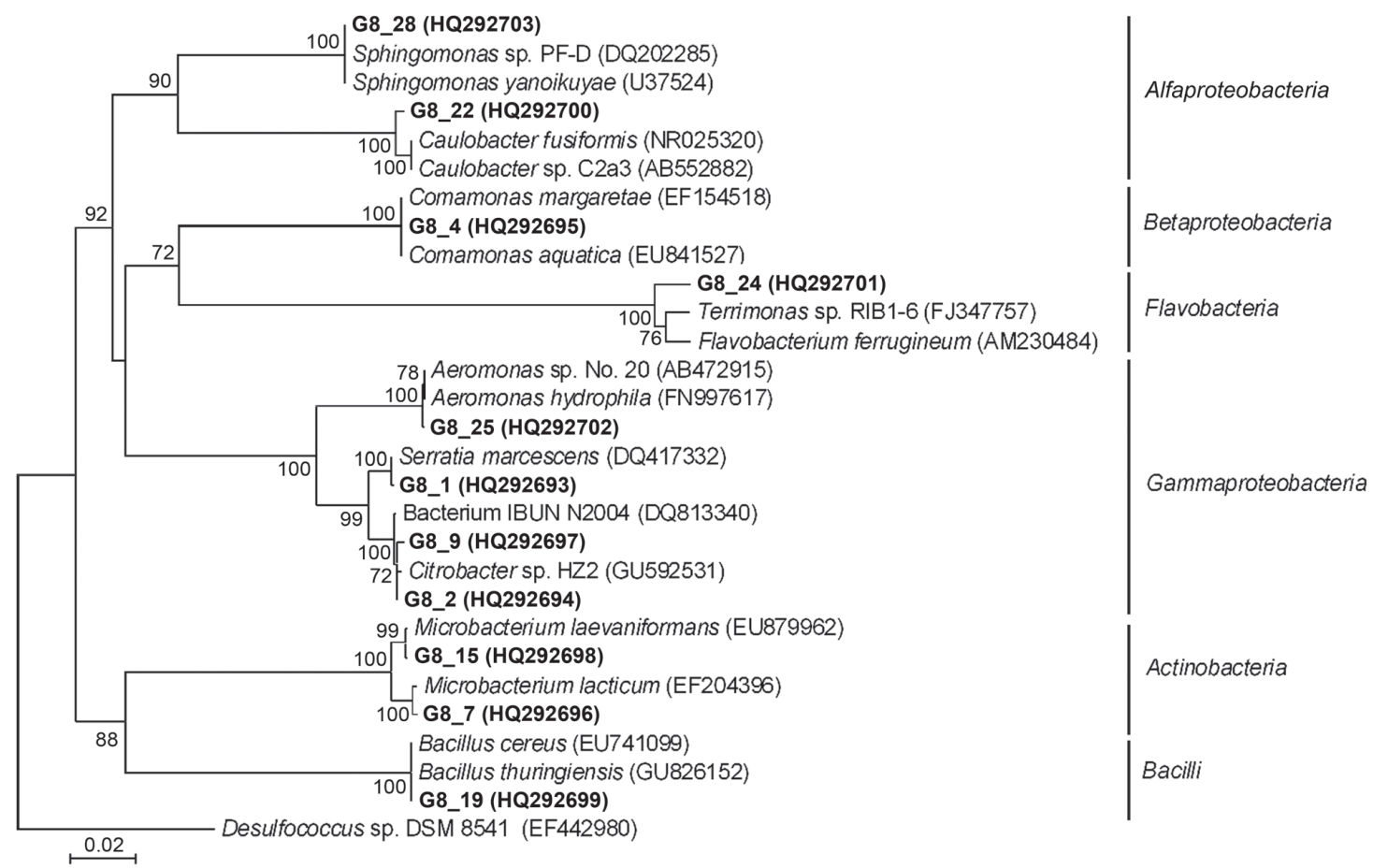

Fig. 2. Phylogenetic tree generated by the neighbor-joining method showing the phylogenetic relationships among bacteria isolated from biomass utilizing the glycerol and their nearest neighbors. Desulfococcus sp. DSM8541 was selected as the outgroup. The numbers on the branches refer to bootstrap values for 1,000 times. Accession numbers are given in parentheses. 


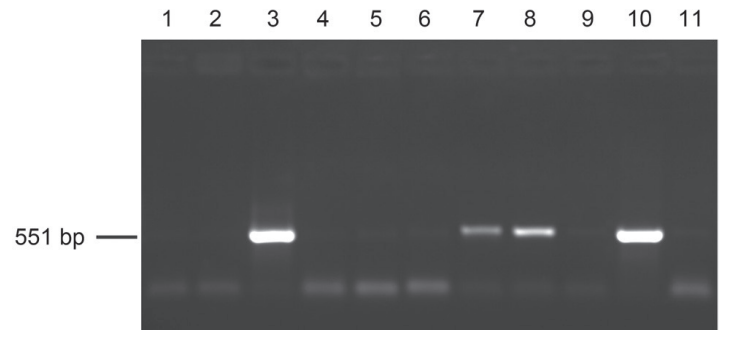

Fig. 3. Detection of PHAs producers by amplification of fragments of PHA synthase genes using PCR primers developed by Romo et al. (2007). A positive signal (551 bp) was recorded for strains G8_4 (lane 3), G8_19 (lane 7), G8_22 (lane 8), G8_25 characterized in Table I.

To detect microorganisms possessing the ability of polyhydroxyalkanoates synthesis, PCR was performed with a primer recognizing both class I and II PHA synthase genes (Romo et al., 2007). The amplification gave a positive signal in 4 of the isolates, corresponding to Comamonas sp. (G8_4), Bacillus sp. (G8_19), Caulobacter sp. (G8_22), and Aeromonas sp. (G8_25) (Table I). All the obtained PCR amplicons possessed the expected length of $551 \mathrm{bp}$ (Fig. 3).

\section{Discussion}

One of the best known methods for the natural selection of microorganisms synthesizing PHAs is the enrichment of microorganisms from activated sludge. The batch-wise feeding that is applied in such a process results in periods with carbon source excess (feast phase) and periods without substrate (famine phase). Microorganisms that have the ability to accumulate carbon and energy in the form of PHAs and then use them in degradation will have ascendancy over others that are not able to survive the famine phase. Thus, this type of process favors the PHAs-accumulating microorganisms (Reis et al., 2003; Ciesielski et al., 2009). In this study, the selection of a culture with a high capacity for PHAs accumulation was obtained by separating the SBR cycles. During the PHAs synthesis phase, polymer synthesis was induced under nitrogen limited-conditions and during the biomass regeneration phase, when glycerine feeding was stopped, enabling the consumption of PHAs in the endogenous metabolism. Intracellular PHAs content was comparable to that observed by others using mixed cultures selected in an aerobic dynamic feeding system (ADF) (Albuquerque et al., 2007), and slightly lower in comparison with pure cultures of Cupriavidus necator DSM 545 (Cavalheiro et al., 2009) cultivated on waste glycerol. The observed PHAs yield coefficient on substrate (COD) at a level of $0.30 \mathrm{~g} \mathrm{PHA} / \mathrm{g}$ COD, obtained in this research was comparable to the results obtained during PHAs pro- duction using Alcaligenes latus, when a level of $0.23 \mathrm{~g}$ $\mathrm{PHB} / \mathrm{g}$ mixture of methanol and glycerol was reached (Braunegg et al., 1999).

Most probably, owing to periodical changes of substrate levels as well as the presence of many physiologically specialized microorganisms, the SBR process could promote plasmid-mediated transfer of genes responsible for PHAs synthesis and accumulation. Despite the increasing number of researches focusing on PHAs production by mixed cultures, there is still a lack of detailed information concerning the microorganisms involved in these biopolymers' synthesis. In this study, the bacteria in an undefined microbial community fed waste glycerol were subjected to identification. Among twenty isolated strains, eleven belonged to unique phylogenetic genera. They belonged to six different phylogenetic lineages (Fig. 2), thus it could be postulated that the analyzed bacterial community is highly diversified. The presence of microorganisms having the genetic potential of PHAs synthesis was determined by PCR detection. For this task, the universal PCR primers elaborated by Romo et al. (2007) were applied. Among all the isolated strains, four of them gave a positive signal. The discovered PHA-producers were classified as belonging to the genera Comamonas (G8_4), Bacillus (G8_19), Caulobacter (G8_22), and Aeromonas (G8_25) (Table I). All of them were previously described as PHAs producers (Lee, 1996). Moreover, all four strains are known as being responsible for short-chain-length-PHAs synthesis. Gas chromatography analysis showed that only polyhydroxybutyrate was produced by the microbial community, which suggests that the isolated strains could be the main PHAs producers. Unfortunately, the performed analysis does not allow us to point out which of them play the main role in this process. This may be achieved by culturing these strains independently in similar technological conditions.

In our previous paper (Ciesielski et al., 2010), the structure of the microbial community utilizing waste glycerol was analyzed. The experiment performed in a sequencing batch reactor under aerobic dynamic feeding conditions allowed for the selection of only one polyhydroxybutyrate producing bacterium belonging to the species Rhodobacter. Although in this process only short-chain-length PHA was detected, three different Pseudomonas species, known as medium-chainlength PHA producers were also detected. Generally, the microbial structure in the mentioned study, exhibited by the culture-based approach, was different from the microbial structure demonstrated here. Because the inoculum was taken from the same source, and the applied substrate was crude glycerol in both cases, it could be presumed that the observed difference could have resulted from the different feeding regime. 
In conclusion, we demonstrated that microbial communities cultured towards PHAs production are created by diversified microorganisms belonging to different phylogenetical lineages. Among eleven unique strains, four had the genetic potential of PHAs synthesis, thus it could be concluded that the microbial community enriched from activated sludge is a promising source of PHAs producers. In the next step, the discovered bacterial strains should be tested in order to estimate their ability of PHAs formation during cultivation. In such an approach, waste glycerine should be examined as a carbon source, which will create an opportunity to develop an economically viable system for utilizing waste from the biodiesel industry.

\section{Acknowledgments}

The study was financed under project no. N207 069 31/3394 of the Polish Ministry of Science and Higher Education.

\section{Literature}

Albuquerque M.G.E., M. Eiroa, C. Torres, B.R. Nunes and M.A.M. Reis. 2007. Strategies for the development of a side stream process for polyhydroxyalkanoate (PHA) production from sugar cane molasses. J. Biotechnol. 130: 411-421.

Anderson A.J. and E.A. Dawes. 1990. Occurence, metabolism, metabolic role and industrial uses of bacterial polyhydroxyalkanoates. Microbiol. Rev. 54: 450-472.

Ataei S.A., E. Vasheghani-Farahani, S.A. Shojaosadati and H. Abdol Tehrani. 2008. Isolation of PHA Producing Bacteria from Date Syrup Waste. Macromol. Symp. 269: 11-16.

Braunegg G., K. Genser, R. Bona, G. Haage, F. Schellauf and E. Winkler. 1999. Production of PHAs from agricultural waste material. Macromol. Symp. 144: 375-383.

Cavalheiro J.M.B.T., M.C.M.D. de Almeida, C. Grandfils and M.M.R. da Fonseca. 2009. Poly(3-hydroxybutyrate) production by Cupriavidus necator using waste glycerol. Process Biochem. 44: 509-515.

Ciesielski S., A. Cydzik-Kwiatkowska, T. Pokoj and E. Klimiuk. 2006. Molecular detection and diversity of medium-chain-length polyhydroxyalkanoates producing bacteria enriched from activated sludge. J. Appl. Microbiol. 101:190-199.

Ciesielski S., E. Klimiuk, J. Możejko, E. Nowakowska and T. Pokoj. 2009. Changes in microbial community structures during adaptation towards polyhydroxyalkanoates production. Pol. J. Microbiol. 58: 131-139.

Ciesielski S., T. Pokoj and E. Klimiuk. 2010. Cultivation-dependent and -independent characterization of microbial community producing polyhydroxyalkanoates from raw-glycerol. J. Microbiol. Biotechnol. 20: 853-861.

Comeau Y., K.J. Hall and W.K. Oldham. 1988. Determination of poly- $\beta$-hydroxybutyrate and poly- $\beta$-hydroxyvalerate in activated sludge by gas-liquid chromatography. Appl. Environ. Microb. 54: 2325-2327.

da Silva G.P., M. Mack and J. Contiero. 2009. Glycerol: a promising and abundant carbon source for industrial microbiology. Biotechnol. Adv. 27: 30-39.
Dias J. M. L., P.C. Lemos, L.S. Serafim, C. Oliveira, M. Eiroa, M.G.E. Albuquerque, A.M. Ramos, R. Oliveira and M.A.M. Reis. 2006. Recent advances in polyhydroxyalkanoate production by mixed aerobic cultures: From the substrate to the final product. Macromol. Biosci. 6: 885-906.

Ibrahim M.H.A. and A. Steinbüchel. 2009. Poly(3-hydroxybutyrate) production from glycerol by Zobellella denitrificans MW1 via high-cell-density fed-batch fermentation and simplified solvent extraction. Appl. Environ. Microbiol. 75: 6222-6231.

Ibrahim M.H.A. and A. Steinbïchel. 2010. Zobellella denitrificans strain MW1, a newly isolated bacterium suitable for poly(3hydroxybutyrate) production from glycerol. J. Appl. Microbiol. 108: 214-225.

Kumar S., K. Tamura, I.B. Jakobsen and M. Nei. 2001. MEGA2: Molecular Evolutionary Genetics Analysis software. Bioinformatics 17: $1244-1245$.

Kumbhakar S., P.K. Singh and A.S. Vidyarthi. 2012. Screening of root nodule bacteria for the production of polyhydroxyalkanoate (PHA) and the study of parameters influencing the PHA accumulation. Afr. J. Biotechnol. 11: 7934-7946.

Lee S.Y. 1996. Bacterial polyhydroxyalkanoates. Biotechnol. Bioeng. 49: 1-14.

López-Cortés A., L.A. Lanz and M.J.Q. Garcia. 2008. Screening and isolation of $\mathrm{PHB}$-producing bacteria in a polluted marine microbial mat. Microb. Ecol. 56: 112-120.

Miskin I.P., P. Farrimond and I.M. Head. 1999. Identification of novel bacterial lineages as active members of microbial populations in a freshwater sediment using a rapid RNA extraction procedure and RT-PCR. Microbiology 145: 1977-1987.

Oehmen, A., B. Keller-Lehmann, R. J. Zeng, Z. Yuan and J. Keller. 2005. Optimisation of poly- $\beta$-hydroxyalkanoate analysis using gas chromatography for enhanced biological phosphorus removal systems. J. Chromatogr. A 1070: 131-136.

Pisco A. R., S. Bengtsson, A. Werker, M.A.M. Reis and P.C. Lemos. 2009. Community structure evolution and enrichment of glycogenaccumulating organisms producing polyhydroxyalkanoates from fermented molasses. Appl. Environ. Microbiol. 75: 4676-4686.

Reis M.A.M., L.S. Serafim, P.C. Lemos, A.M. Ramos, F.R. Aguiar and M.C.M. van Loosdrecht. 2003. Production of polyhydroxyalkanoates by mixed microbial cultures. Bioprocess Biosyst. Eng. 25: 377-385.

Romo D.M.R., M.V. Grosso, N.C.M. Solano and D.M. Casta. 2007. A most effective method for selecting a broad range of short and medium-chain-length polyhydroxyalkanoates producing microorganisms. Electron. J. Biotechnol. 10: 348-357.

Satoh H., Y. Iwamoto, T. Mino and T. Matsuo. 1998. Activated sludge as a possible source of biodegradable plastics. Wat. Sci. Tech. 38: 103-109.

Serafim L.S., P.C. Lemos, R. Oliveira and M.A.M. Reis. 2004 Optimization of polyhydroxybutyrate production by mixed cultures submitted to aerobic dynamic feeding conditions. Biotechnol. Bioeng. 87: 145-160.

Stackebrandt E. and B.M. Goebel. 1994. A place for DNA-DNA reassociation and $16 \mathrm{~S}$ rRNA sequence analysis in the present species definition in bacteriology. Int. J. Syst. Bacteriol. 44: 846-849.

Thompson J. D., D.G. Higgins and T.J. Gibson. 1994. CLUSTAL $\mathrm{W}$ : Improving the sensitivity of progressive multiple sequence alignment through sequence weighting, positions specific penalties and weight matrix choice. Nucleic Acids Res. 22: 4673-4680.

Van Loosdrecht M.C.M., M.A. Pot and J.J. Heijnen. 1997. Importance of bacterial storage polymers in bioprocesses. Wat. Sci. Tech. 35: 41-47. 